\title{
4G/LTE Channel Quality Reference Signal Trace Data Set
}

\author{
Britta Meixner \\ Centrum Wiskunde \& Informatica, \\ Tiledmedia B.V. \\ Amsterdam, The Netherlands \\ britta.meixner@cwi.nl
}

\author{
Jan Willem Kleinrouweler \\ Centrum Wiskunde \& Informatica \\ Amsterdam, The Netherlands \\ j.w.m.kleinrouweler@cwi.nl
}

\author{
Pablo Cesar \\ Centrum Wiskunde \& Informatica, \\ TU Delft \\ Amsterdam, The Netherlands \\ P.S.Cesar@cwi.nl
}

\begin{abstract}
Mobile networks, especially LTE networks, are used more and more for high-bandwidth services like multimedia or video streams. The quality of the data connection plays a major role in the perceived quality of a service. Videos may be presented in a low quality or experience a lot of stalling events, when the connection is too slow to buffer the next frames for playback. So far, no publicly available data set exists that has a larger number of LTE network traces and can be used for deeper analysis. In this data set, we provide 546 traces of 5 minutes each with a sample rate of $100 \mathrm{~ms}$. Thereof 377 traces are pure LTE data. We furthermore provide an Android app to gather further traces as well as R scripts to clean, sort, and analyze the data.
\end{abstract}

\section{CCS CONCEPTS}

- Networks $\rightarrow$ Network measurement;

\section{KEYWORDS}

LTE data, RSRP, RSRQ, ASU, DBM, Speed, mobile phone

\section{ACM Reference Format:}

Britta Meixner, Jan Willem Kleinrouweler, and Pablo Cesar. 2018. 4G/LTE Channel Quality Reference Signal Trace Data Set. In MMSys'18: 9th ACM Multimedia Systems Conference, June 12-15, 2018, Amsterdam, Netherlands. ACM, New York, NY, USA, 6 pages. https://doi.org/10.1145/3204949.3208132

\section{INTRODUCTION}

Many people use mobile networks for their internet access. Most of them rely on mobile internet when on the go, but also the number of people relying on their mobile device for internet access using high data volume contracts increases [17][19]. Data intensive services, such as video streaming or other multimedia applications [18][5], have found their way to mobile devices. However, mobile networks pose a much more challenging networking environment compared to their wired counterparts. Throughput from base station to end-user device is a function of radio channel conditions and the current load of the cell. As a result, mobile connections show large throughput variations that have to be dealt with to provide a smooth networking experience. The availability of mobile

Permission to make digital or hard copies of all or part of this work for personal or classroom use is granted without fee provided that copies are not made or distributed for profit or commercial advantage and that copies bear this notice and the full citation on the first page. Copyrights for components of this work owned by others than the author(s) must be honored. Abstracting with credit is permitted. To copy otherwise, or republish, to post on servers or to redistribute to lists, requires prior specific permission and/or a fee. Request permissions from permissions@acm.org.

MMSys'18, June 12-15, 2018, Amsterdam, Netherlands

(C) 2018 Copyright held by the owner/author(s). Publication rights licensed to Association for Computing Machinery.

ACM ISBN 978-1-4503-5192-8/18/06 . \$ \$15.00

https://doi.org/10.1145/3204949.3208132 network traces can be of great value when developing new mobile applications or running experiments in a mobile context.

In this data set, we present a large set of traces that describe the radio channel conditions between base station and end-user device. The traces can be used to realistically model and simulate a mobile networking environment. We measured LTE signal strength values, including Signal Strength, Reference Signal Received Power (RSRP) and Reference Signal Received Quality (RSRQ). These indicators provide a detailed insight into the channel quality between LTE eNodeB and User Equipment (UE). By measuring signal strength indicators instead of throughput, we provide the users of our data set the flexibility to evaluate solutions ranging from single node applications to quality of service/scheduling algorithms in LTE base stations.

The LTE traces consist of signal strength indicators that we collected using two LG Nexus $5 \mathrm{Xs}^{1}$. Our data set and its related materials provide the following contributions:

- A data set consisting of 377 LTE traces, summing up to 1125919 data samples. Additional 169 traces with mixed LTE and non-LTE signal strength indicators. Each trace has a duration of 5 minutes and a sample-rate of $100 \mathrm{~ms}$.

- Annotated traces of different environments and at different speeds.

- Traces for two network providers and in three countries.

- R scripts for cleaning, sorting, data analysis, and data visualization.

To the best of our knowledge, such an extensive data set does not exist for LTE networks. The proportions of this data set open doors for analysis of frequently occurring patterns. Such patterns can be used to improve network predictions. This makes our data set useful for both application developers and networking researchers.

The remainder of this work is structured as follows: First, we give an overview of related work, especially similar data sets in the area of LTE networks, in Section 2. We then describe the software that was used for recording the data consisting of an Android app and a Server app (Section 3). In Section 4, we describe how the data was recorded. After that, we provide details about our data set in Section 5. We provide three example use cases of our data set in Section 6. The work ends with a conclusion (Section 7).

\section{RELATED WORK}

A search for a mobile network data set, especially for cellular networks, reveals data sets for varying use cases. One area are wireless antennas, where data sets are used for performance improvements, as for example described by Amiri Sani et al. [1][15] and Anderson et al. [2][3]. Another area are measurements in WiMAX networks,

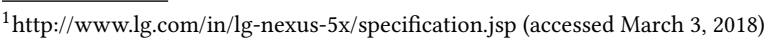


especially for video streaming applications. Fund et al. collect measurements from DASH and WebRTC implementations while moving at walking speeds through an 802.16e WiMAX network [10][9]. Besides information about the transmitted videos (DASH and WebRTC), the received signal strength indicator (RSSI), carrier to interference plus noise ratio (CINR), and frequency are collected by a WiMAX logger. Furthermore, latitude, longitude, altitude, speed, time, and fix mode (2D or 3D) are recorded via a GPS logger. This paper gives hints on useful data which was taken into consideration while designing our recording tool.

Several data sets and tools can be found for $3 \mathrm{G}$ networks. Yoon et al. introduce WiScape, which captures data to provide an accurate performance characterization of the $3 \mathrm{G}$ networks [23][16][22]. Di Francesco et al. gathered a data set containing census information, cellular infrastructure deployment, and cellular data demand [8]. They differentiate between urban and rural areas. For the networks, they collect coordinates, power class, technology $(2 \mathrm{G} / 3 \mathrm{G})$ and mobile phone operator. They furthermore look into specifics of data transfers. Vallina-Rodriguez et al. describe an open source tool for performing $3 \mathrm{G}$ network analysis on mobile phones, they however do not provide a data set with it [20]. We carefully analyzed these tools for collected data and recording techniques, and implemented the lessons learned into our tool.

Taking a look at existing data sets and tools for 4G/LTE data, only data sets for very limited use cases could be found. Two data sets were found for multi-path TCP analysis which mainly focuses on the analysis of routing information. De Coninck et al. investigate multi-path TCP traffic from smartphones for wireless and cellular networks [7][6]. Their methodology is based on the detection of handovers. Nikravesh et al. perform multi-path TCP measurements on WiFi and cellular networks by performing three tasks: active and passive measurements, and data upload [14]. They collect TCP/IP header data as well as other performance data from the individual tests. These data are too limited for our purpose as we are interested in network performance, not on routing. Huang et al. look into upand down-link throughput as well as power usage of LTE networks compared to $3 \mathrm{G}$ and WiFi networks [12]. While the tools used for data collection are publicly available, the extensive data used for their analysis could not be found. Bergal et al. performed point measurements of LTE signals on a university campus. Devices are placed on pre-determined given spots, then, LTE signal measurements were taken [4]. This results in a data set for a very limited geographical area. Furthermore, devices did not move during measurements. Hosseini et al. look into the bandwidth fluctuation for connection speeds from low speed satellite links to $4 \mathrm{G}$ networks on paths between two hospitals in Illinois [11]. They collect data from four major mobile carriers in the US. Their data set consists of data gathered by continuous sampling of communication bandwidths (download and upload rates), GPS coordinates, GPS accuracy, altitude, and vehicle velocity and bearing. This data set is also limited to a small geographical area. However, all data sets that we could find looked into down- and upload rates. None of them provides data on the channel quality reference signals, which are needed for deeper analysis of the network and modeling of application behavior.

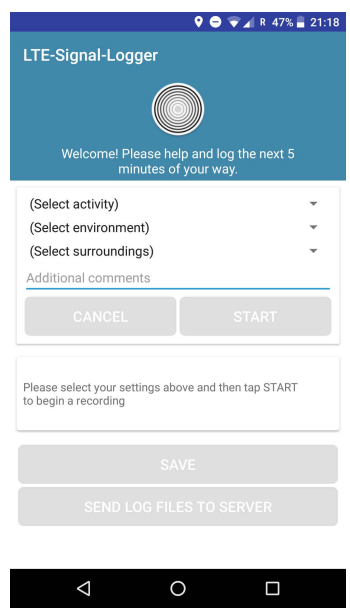

(a) Start screen without any selections and deactivated buttons

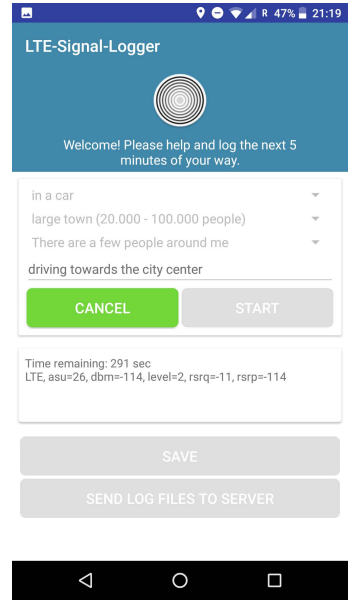

(c) Screen during recording with disabled meta-data spinners and countdown

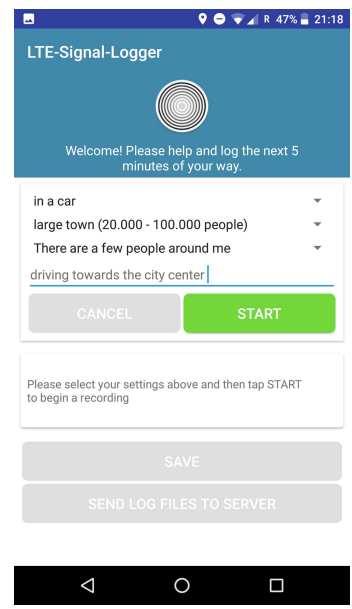

(b) Screen with selected metadata and activated start button

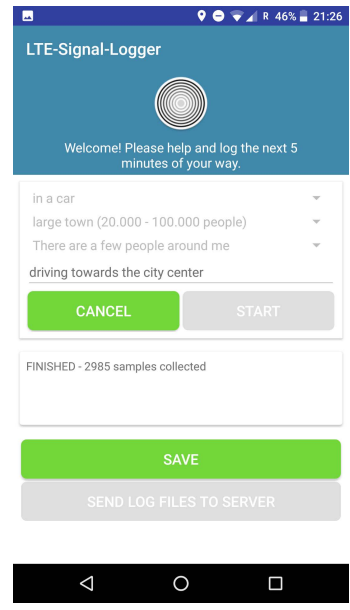

(d) Screen after recording with a completed trace (can be saved or deleted)
Figure 1: Illustration of the network data tracing app.

\section{SOFTWARE FOR DATA COLLECTION}

To collect our data, we implemented a smart-phone app. This app was then used to record traces from mobile networks. Each set of traces was saved locally and then transmitted to our data collection server. Code and data set are provided here: https://doi.org/10.5281/ zenodo.1220256.

\subsection{Android App}

We developed an Android app to capture meta-data and network traces. The app uses the Telephony API from Android SDK 26. The user interface provides spinner fields for setting meta-data like the area where the trace was recorded, the activity during recording the trace, and the environment of recording the trace. For area, the user could select from the following values: 
- I am (almost) alone

- There are a few people around me

- I am in a small crowd (city center, etc.)

- I am in a big crowd (stadion, etc.)

To allow an easier decision on the environment, we use the definitions close to [21] and added numbers for clarification. Users can pick between the following environments:

- middle of nowhere

- village (1.000 people or less)

- town (1.000 - 20.000 people)

- large town (20.000 - 100.000 people)

- city (100.000 - 300.000 people)

- large city (300.000 - 1.000 .000 people)

- metropolis (more than 1.000.000 people)

We furthermore wanted to get an estimation of the moving speed and moving pattern. In addition to area and environment, we ask during which activity the trace is recorded:
- sitting/standing
- walking
- running
- biking
- biking (fast)
- in a car
- in a bus
- in a train
- on a plane

We furthermore provide a free text field, which allows the user to add additional comments or circumstances which cannot be covered by the three spinners. Before recording a trace can be started, area, activity, and environment have to be set, otherwise the start button is disabled (see Figure 1a). Once area, activity, and environment are set, the start button is enabled (see Figure $1 \mathrm{~b}$ ) and recording a trace can be started. During recording a trace, the counter counts downwards to zero (see Figure 1c). At the end of recording, the user can chose to save or discard the recorded data (see Figure 1d).

To allow recording traces in the background, we implemented the data collection as a service (extends Service). However, Android switches into power saving mode when the screen is turned off, which means, that less updates on the signal strength (onSignalStrengthsChanged(SignalStrength sStrength)) are provided. For that reason, the screen has to be kept on during recording a trace.

\subsection{Server}

After recording a trace, the user has the ability to upload trace files to a server, either as a single trace or as a batch of traces on a later moment. Our server provides a simple HTTP end-point that our application uses for uploading the traces. The server stores the traces files on disk, but also comes with the option to upload the trace to a Dropbox folder. The source code of both, the mobile application and the server, are provided with the data set.

\section{DATA RECORDING}

We used 4G LTE SIM Cards from the following cellular carriers: T-Mobile (US), T-Mobile (Germany), and Vodafone (Netherlands).
We recorded data mainly in California (USA), in Bavaria and Berlin (Germany), and all over the Netherlands. For each trace, meta-data was selected in the app. Then, recording the trace was started and it was made sure that the screen did not turn off by setting the screen-off-timer to 10 minutes. After the data was collected, the trace was saved locally and then uploaded to the server.

Traces were recorded during all activities mentioned in the previous section. However, stops due to traffic (for example in a car at a red light) or due to the type of transport (for example bus/train stops to pick up people) could not be avoided. Nevertheless, this means that the traces represent realistic conditions. While we were interested in LTE data for our research, in some cases the network type dropped out of $4 \mathrm{G}$ to lower bandwidths. We recorded values for $3 \mathrm{G}$ data as well as basic values for slower networks. Depending on the phone and chip or because of malfunctions during recording, the API may not provide all values. In this case, Integer.MIN_VALUE is recorded in the corresponding field.

\section{DATA SET STRUCTURE}

We describe our data set from two points of view - on the one hand we show how a single file is structured, on the other hand we show how traces are distributed over different settings.

\subsection{File Structure}

Each file consists of a header and a data section. The header contains meta-data for the whole trace. A list of saved variables, their source of input (app/user), and their data-type and format are listed in Table 1. For each file, the start and end of the trace, the location where the trace was started, as well as phone model and network provider are queried from the Android-API. In addition to that, the activity while recording the trace, the area, and the environment, as well as a note are saved from user input via the GUI.

The data section consists of on average 2987 data rows representing a 5 minute trace in 100 millisecond steps. Slight variations in the number of data rows are a result of background processes on the Android phones which could not be avoided. However, these can also be observed in real use cases while watching videos on smart-phones. As can be seen in Table 2, the standard deviation

Table 1: Header data of a trace file

\begin{tabular}{|c|c|c|}
\hline $\begin{array}{l}\text { Variable } \\
\text { ju }\end{array}$ & $\begin{array}{l}\text { input } \\
\text { from }\end{array}$ & datatype/format \\
\hline recordingStart & app & time (mm:ss.SSS) \\
\hline recordingEnd & app & $\begin{array}{l}\text { datetime (format: } \\
\text { (yyyy-mm-dd hh:mm:ss.SSS)) }\end{array}$ \\
\hline recordingActivity & user & string \\
\hline recordingArea & user & string \\
\hline recordingEnvironment & user & string \\
\hline note & user & string \\
\hline city & app & string \\
\hline region & app & string \\
\hline country & app & string \\
\hline phoneModel & app & string \\
\hline networkProvider & app & string \\
\hline
\end{tabular}


Table 2: Data section of a trace file

\begin{tabular}{lllllll}
\hline Variable & datatype & $\min$ & $\max$ & mean & median & sd \\
\hline row & int & 1 & 2993 & 2986.523 & 2987 & 1.8013 \\
currentTime & datetime & \multicolumn{5}{c}{ format: YYYY-MM-DD HH:MM:SS.mmm } \\
longitude & double & -122.4154 & 13.46374 & -7.6503 & 12.05898 & 44.8977 \\
latitude & double & 37.34431 & 52.55427 & 48.4865 & 48.91395 & 4.6065 \\
speed & double & 0 & 44.64 & 13.9471 & 10.37255 & 13.1583 \\
networkType & string & networkType $\in$ \{HSPA, HSPAP, LTE, UNKNOWN, NONE, EDGE, UMTS, GPRS $\}$ \\
mcc & int & \multicolumn{5}{c}{ see Table 3 } \\
mnc & int & \multicolumn{5}{c}{ see Table 3 } \\
asu & int & 0 & & 44.8105 & 44 & 12.2644 \\
power & double & $4.940656 e-324$ & $1 \mathrm{e}-08$ & 0 & $1 \mathrm{e}-12$ & 0 \\
dbm & dbm & -140 & -52 & -95.1895 & -96 & 12.2644 \\
level & int & 1 & 4 & 3.2363 & 3 & 0.8775 \\
rsrq & int & -20 & -3 & -9.2718 & -9 & 2.9777 \\
rsrp & int & -140 & -52 & -95.1895 & -96 & 12.2644 \\
tac & int & 265 & 65535 & 21295.4 & 22006 & 14994.07 \\
enb & int & 60 & 16777215 & 106827 & 100125 & 450037.8 \\
eci & int & -1 & 230424321 & 24273109 & 25632011 & 15055960 \\
pci & int & 0 & 501 & 246.3232 & 250 & 134.0798 \\
earfcn & int & 1025 & 6400 & 3716.491 & 2300 & 2291.609 \\
\hline
\end{tabular}

the number of rows is 1.8013 , which makes the files comparable. Table 2 furthermore gives an overview of the recorded values. We list all relevant fields for the LTE standard with their data-type. However, not all values are provided by the Android-API using a Nexus 5X. In our case, these were band, rssnr, rssi, nid, cqi, lcid, ta, snr, and signalStrengt $h^{2}$. We provide recorded minimum, maximum, mean, median, and standard deviation for each provided value (after removing errors resulting in Integer.MIN_VALUE falsifying the results).

\subsection{Trace Metadata}

We collected 377 traces with only LTE data, those are used in the remainder of this paper. Non-LTE traces were moved to extra folders and are provided for the sake of completeness. Accordingly, the data set contains overall 546 traces.

We did traces in mainly three countries and for two network providers. From the 377 traces, 120 were done in the Netherlands, 2 in Italy, 205 in Germany, and 50 in the US. We used three different SIM cards. One was a German T-Mobile SIM card, this was used for recording 210 traces. A Dutch Vodafone SIM card was used for recording 117 traces, and a US T-Mobile SIM card was used for recording 50 traces. An overview of countries and network providers can be found in Table 3 (as listed in [13]).

For the LTE traces, the following area settings were selected: I am (almost) alone (152). There are a few people around me (204). I am in a small crowd (city center, etc.) (7). I am in a big crowd (stadion, etc.) (14). For the environment settings we gathered: middle of nowhere (131), village (17), town (23), large town (92), city (21), large city (90), and metropolis (3). Taking a look at the activities indicating the

\footnotetext{
${ }^{2}$ What values are provided depends on the chip used in the phone, it may also vary from phone model to phone model and between API levels. In case more traces are recorded, some of the values may become available.
}

Table 3: Traces by country (MMC) and network provider (MNC)

\begin{tabular}{llllll}
\hline MCC & country & MNC & network provider & \#samples & \#rec. \\
\hline 204 & NL & 4 & Vodafone Libertel & 143278 & 48 \\
204 & NL & 16 & T-Mobile B.V. & 214764 & 72 \\
222 & IT & 10 & Vodafone & 5970 & 2 \\
262 & DE & 1 & T-mobile/Telekom & 411755 & 138 \\
262 & DE & 2 & Vodafone D2 & 200127 & 67 \\
310 & US & 260 & T-Mobile & 149218 & 50 \\
\hline
\end{tabular}

speed of movement, we have the following results: sitting/standing (37), walking (50), running (2), biking (2), in a car (261), in a bus (19), in a train (6), and none for biking (fast) and on a plane.

Figure 2 shows a summary of the data by area, activity, and environment. To give a better overview, traces with low numbers of samples are not displayed and related factors are shown as one (eg. village and middle of nowhere are summarized as rural). It can be noticed that most of the traces are recorded in a car or while walking. While traces during standing were recorded in most environments, only few (valid) traces were recorded in buses.

\subsection{Trace Data}

As our data set contains a huge number of variables, we hereafter take a closer look at two examplary distinct ones, RSRQ and speed. Taking a look at the RSRQ values, it can be noticed, that the data is not normally distributed (see Figure 3d). The data shows a negative skew which in case of RSRQ means that the signal quality was better than average ( -3 is very high quality, -20 very low quality). Figures $3 \mathrm{a}, 3 \mathrm{~b}$, and $3 \mathrm{c}$ show box-plots with the lower and upper 


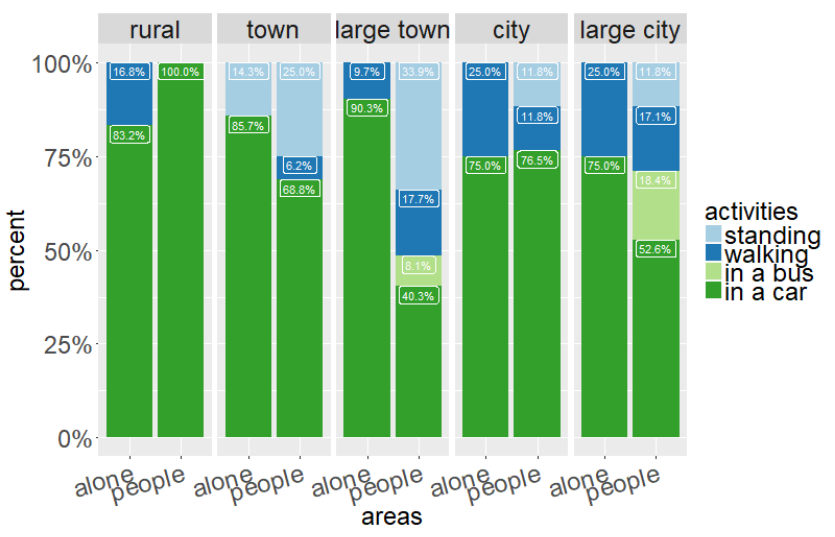

Figure 2: Distribution of the traces by area, activity, and environment.

quartile at $25 \%$ and $75 \%$ percent. It can be noticed that only slight differences exist between the factors in different groupings.

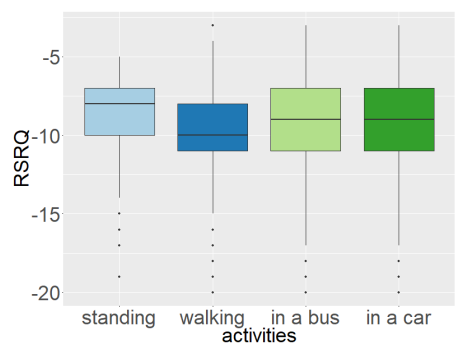

(a) RSRQ distribution by activities

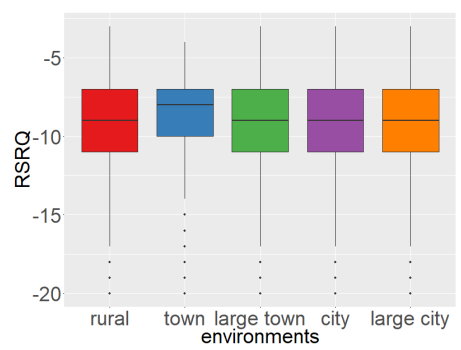

(c) RSRQ distribution by environments

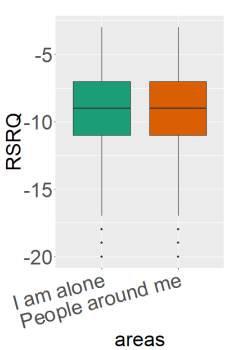

(b) RSRQ distribution by areas

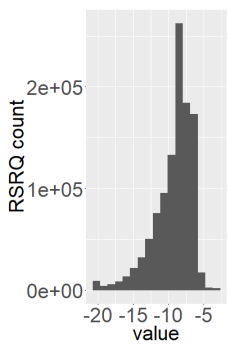

(d) Histogram for all RSRQ values
Figure 3: Visualization of RSRQ data by activities, areas, and environments.

Figure 4 shows the speed distributions that were measured while recording a trace. It can be noticed that the bus has a comparably low speed, where the low quartile is at zero resulting from stops at bus stops. The median for driving a car is around $80 \mathrm{~km} / \mathrm{h}$. While we tried to keep the phone as steady as possible, quick movements caused some outliers. Those could be avoided in buses and cars, where recording a trace was done sitting.

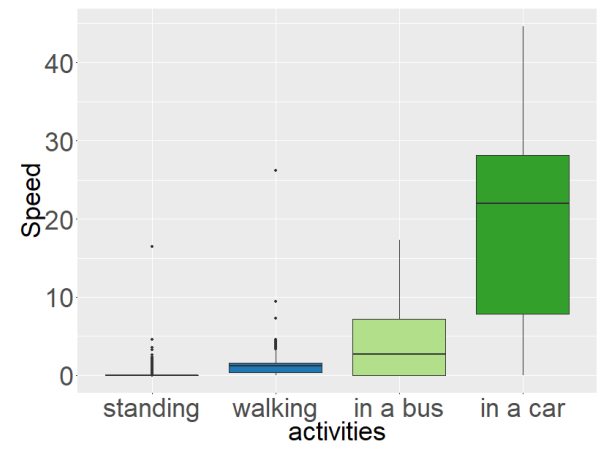

Figure 4: Distribution of the traces by speed (in $\mathrm{m} / \mathrm{s}$ ) and environment.

\section{USE CASES FOR THE DATA SET}

With this data set, we aim to support realistic simulations of the LTE channel conditions between base station and client device. In this section, we provide three use cases how our data set can be applied.

QoS for video streaming. We use this data set ourselves to implement a Quality of Service (QoS) solution for adaptive video streaming. Depending on the channel conditions, a modulation scheme with more or less data redundancy is used. The cost (as in network LTE resource blocks) to guarantee a certain throughput to a video client therefore strongly depends on the quality of the LTE signal. When channel conditions are good, we allow video clients to grow their buffer. When channel quality decreases, clients can rely on their buffer. We use the RSRQ values to estimate channel conditions between base station and client. We used the RSRQ values in this data set to predict how channel conditions will evolve. Figure 5 shows how often the reference signal changes from one level to another. Using these predictions, we were able to compute the optimal buffering strategy. With this strategy we are also able to reduce the overall network costs for video streaming.

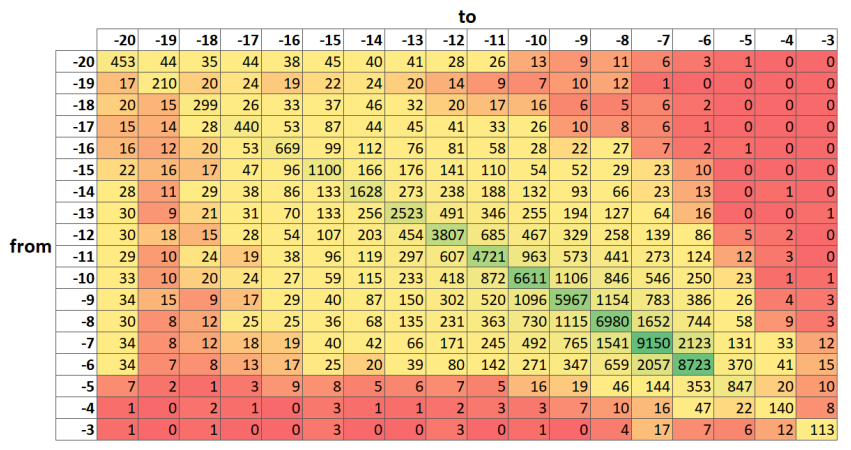

Figure 5: Counting RSRQ level changes.

Correlations and Trends. We used the data for a first deeper analysis towards trend prediction and correlation analysis. On the one hand, we tried to predict future trends in the RSRQ values using smoothing curves and their first and second deviations. With the 
deviations we could calculate upward and downward trends. On the other hand, we tried to find correlations in our data set. We expected a correlation between speed and LTE signal quality, but the correlation analysis did not confirm this assumption. This shows, that at this point in time, speed as reported by the mobile device cannot be used to further improve the QoS for video streaming as described in the previous paragraph.

Mobile Edge Cloud handovers. Mobile Edge Clouds (MEC) currently receive a lot of attention in research. MEC provides computing resources close to the client, and thus greatly reducing the latency between client and cloud. Users are often on the move when using mobile networks. As such, clients will roam between base stations. For MEC to be effective, the computing resources have to travel with the client. Our data set includes to which base station (eNodeB identifier in the enb field, and cell identifier in eci) the client was connected. Combined with GPS locations, our data set can be used to evaluate the flexibility of MEC computing and MEC handovers in many different scenarios.

\section{CONCLUSIONS AND FUTURE WORK}

In this paper, we present a toolkit for recording LTE channel quality reference signals. We furthermore provide a data set with 377 LTE traces containing 1125919 data samples. We collected data in three countries and for two different network providers. With the data set, we show first applications and analysis for our collected data. Our software as well as the data set are publicly available. In order to allow further and deeper analysis of the data, more traces in additional countries and with SIM cards from further network providers are desirable. Due to the fact that we only had a Nexus 5X for recording our traces, we recommend to also use phones from other manufacturers using a wider range of chips. In addition to that, the traces are not evenly distributed over activities, areas, and environment. Accordingly, missing traces should be added to allow more balanced analysis and comparisons.

\section{REFERENCES}

[1] Ardalan Amiri Sani, Lin Zhong, and Ashutosh Sabharwal. 2010. Directional Antenna Diversity for Mobile Devices: Characterizations and Solutions. In Proceedings of the Sixteenth Annual International Conference on Mobile Computing and Networking (MobiCom '10). ACM, New York, NY, USA, 221-232. https://doi.org/10.1145/1859995.1860021

[2] Eric Anderson, Caleb Phillips, Douglas Sicker, and Dirk Grunwald. 2009. Modeling Environmental Effects on Directionality in Wireless Networks. In Proceedings of the 7th International Conference on Modeling and Optimization in Mobile, Ad Hoc, and Wireless Networks (WiOPT'09). IEEE Press, Piscataway, NJ, USA, 564-570. http://dl.acm.org/citation.cfm?id=1715782.1715868

[3] Eric W. Anderson and Caleb Phillips. 2009. CRAWDAD dataset cu/antenna (v 2009-05-08). Downloaded from https://crawdad.org/cu/antenna/20090508. (May 2009). https://doi.org/10.15783/C7VC7V

[4] Eric Bergal, Caleb Philips, and Chingpu Wu. 2012. CRAWDAD dataset cu/lte (v 2012-05-04). Downloaded from https://crawdad.org/cu/lte/20120504. (May 2012). https://doi.org/10.15783/C7KW2N

[5] Cisco Systems, Inc. 2017. Cisco Visual Networking Index: Forecast and Methodology, 2016âĂŞ2021. https://www.cisco.com/c/en/ us/solutions/collateral/service-provider/visual-networking-index-vni/ complete-white-paper-c11-481360.html (accessed February 27, 2018). (2017).

[6] Quentin De Coninck, Matthieu Baerts, Benjamin Hesmans, and Olivier Bonaventure. 2016. CRAWDAD dataset uclouvain/mptcp_smartphone (v. 2016-03-04). Downloaded from https://crawdad.org/uclouvain/mptcp_smartphone/20160304. (March 2016). https://doi.org/10.15783/C7VG6H

[7] Quentin De Coninck, Matthieu Baerts, Benjamin Hesmans, and Olivier Bonaventure. 2016. A First Analysis of Multipath TCP on Smartphones. In 17th International Passive and Active Measurements Conference, Vol. 17. Springer.
[8] P. Di Francesco, F. Malandrino, and L. A. DaSilva. 2017. Assembling and Using a Cellular Dataset for Mobile Network Analysis and Planning. IEEE Transactions on Big Data PP, 99 (2017), 1-1. https://doi.org/10.1109/TBDATA.2017.2734100

[9] Fraida Fund, Cong Wang, Yong Liu, Thanasis Korakis, Michael Zink, and Shivendra Panwar. 2014. CRAWDAD dataset nyupoly/video (v. 2014-05-09). Downloaded from https://crawdad.org/nyupoly/video/20140509. (May 2014). https: //doi.org/10.15783/C7W30R

[10] F. Fund, C. Wang, Y. Liu, T. Korakis, M. Zink, and S. S. Panwar. 2013. Performance of DASH and WebRTC Video Services for Mobile Users. In 2013 20th International Packet Video Workshop. IEEE, 1-8. https://doi.org/10.1109/PV.2013.6691455

[11] Mohammad Hosseini, Yu Jiang, Ali Yekkehkhany, Richard R. Berlin, and Lui Sha. 2017. A Mobile Geo-Communication Dataset for Physiology-Aware DASH in Rural Ambulance Transport. In Proceedings of the 8th ACM on Multimedia Systems Conference (MMSys'17). ACM, New York, NY, USA, 158-163. https: //doi.org/10.1145/3083187.3083211

[12] Junxian Huang, Feng Qian, Alexandre Gerber, Z. Morley Mao, Subhabrata Sen, and Oliver Spatscheck. 2012. A Close Examination of Performance and Power Characteristics of 4G LTE Networks. In Proceedings of the 10th International Conference on Mobile Systems, Applications, and Services (MobiSys '12). ACM, New York, NY, USA, 225-238. https://doi.org/10.1145/2307636.2307658

[13] interactive digital media GmbH. 2013. Mobile Country Codes (MCC) and Mobile Network Codes (MNC). http://www.mcc-mnc.com/ (accessed February 27, 2018). (2013).

[14] Ashkan Nikravesh, Yihua Guo, Feng Qian, Z. Morley Mao, and Subhabrata Sen. 2016. An In-depth Understanding of Multipath TCP on Mobile Devices: Measurement and System Design. In Proceedings of the 22Nd Annual International Conference on Mobile Computing and Networking (MobiCom '16). ACM, New York, NY, USA, 189-201. https://doi.org/10.1145/2973750.2973769

[15] Ardalan Amiri Sani, Lin Zhong, and Ashutosh Sabharwal. 2010. CRAWDAD dataset rice/midas (v. 2010-01-21). Downloaded from https://crawdad.org/rice/ midas/20100121. (Jan. 2010). https://doi.org/10.15783/C7V30F

[16] Sayandeep Sen, Jongwon Yoon, Joshua Hare, Justin Ormont, and Suman Banerjee. 2011. Can They Hear Me Now?: A Case for a Client-assisted Approach to Monitoring Wide-area Wireless Networks. In Proceedings of the 2011 ACM SIGCOMM Conference on Internet Measurement Conference (IMC '11). ACM, New York, NY, USA, 99-116. https://doi.org/10.1145/2068816.2068827

[17] Smart Insights (Marketing Intelligence) Ltd. 2015. Mobile Marketing Statistics compilation - Time Spent per Adult User per Day with Digital Media - USA 2008 - 2015. https://www.smartinsights.com/mobile-marketing/ mobile-marketing-analytics/mobile-marketing-statistics/ (accessed February 27, 2018). (2015).

[18] Statista, Inc. 2014. Online Video Boom Leads to Surge in Data Traffic. https: //www.statista.com/chart/2349/consumer-data-traffic-2013-to-2018/ (accessed February 27, 2018). (2014).

[19] Statista, Inc. 2018. Mobile share of total digital minutes in leading online markets as of May 2017. https://www.statista.com/statistics/266379/ mobile-share- of-digital-minutes-countries/ (accessed February 27, 2018). (2018).

[20] Narseo Vallina-Rodriguez, Andrius Auçinas, Mario Almeida, Yan Grunenberger, Konstantina Papagiannaki, and Jon Crowcroft. 2013. RILAnalyzer: A Comprehensive 3G Monitor on Your Phone. In Proceedings of the 2013 Conference on Internet Measurement Conference (IMC '13). ACM, New York, NY, USA, 257-264. https://doi.org/10.1145/2504730.2504764

[21] Wikipedia, The Free Encyclopedia. 2017. Settlement hierarchy. (2017). https: //en.wikipedia.org/wiki/Settlement hierarchy (accessed December 1, 2017).

[22] Jongwon Yoon, Sayandeep Sen, and Joshua Hare. 2012. CRAWDAD dataset wisc/wiscape (v. 2012-08-03). Downloaded from https://crawdad.org/wisc/ wiscape/20120803. (Aug. 2012). https://doi.org/10.15783/C71C7D

[23] J. Yoon, S. Sen, J. Hare, and S. Banerjee. 2015. WiScape: A Framework for Measuring the Performance of Wide-Area Wireless Networks. IEEE Transactions on Mobile Computing 14, 8 (Aug 2015), 1751-1764. https://doi.org/10.1109/TMC. 2014.2360391 